Pacific Journal of Mathematics

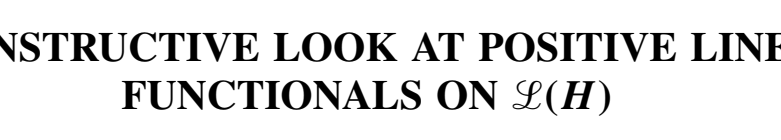




\title{
A CONSTRUCTIVE LOOK AT POSITIVE LINEAR FUNCTIONALS ON $\mathscr{L}(H)$
}

\author{
Douglas S. BRIDGES
}

\begin{abstract}
The main results of this paper characterize countably additive, positive linear functionals on the algebra of bounded operators on a separable Hilbert space, and show how such functionals can be viewed as analogues of positive measures of Bishop-Cheng type. The paper is written entirely within the framework of constructive mathematics.
\end{abstract}

Since the first appearance of Bishop's book [1], constructive techniques have been applied in several areas of analysis, algebra and topology. In this paper, we turn our attention to positive linear functionals on $\mathscr{L}(H)$, the algebra of bounded operators on a serarable Hilbert space $H$.

One reason why a constructive study of these functionals may be of interest is that they are of considerable importance in the vast theory of $C^{*}$-and $W^{*}$-algebras [6], a theory which, in its present form, appears to be highly nonconstructive. Another, and perhaps more important, reason lies in the role played by certain of these functionals in the foundations of physics. Indeed, it is our belief that the development of constructive mathematical foundations for physics may illuminate some of the darker philosophical recesses which present theories seem unable to explore with much success.

Our discussion will rely on some preliminary material on operators of trace class. The constructive problems with this material, which is described in $\S 1$, usually arise from the nonconstructive nature of the least upper bound principle. For example, the distance from a point of $H$ to the range of an operator in $\mathscr{L}(H)$ may not be computable; this means that the classical theory of polar decomposition of an operator has to be modified to one of approximate polar decomposition. Another place where our inability to use the least upper bound principle causes trouble is the proof of completeness of the set of trace class operators with respect to the trace class norm.

In $\S 2$, we give various characterizations of countable additivity of a positive linear functional on the set $\mathscr{A}(H)$ of bounded operators on $H$ for which the adjoint is computable. In $\S 3$, we discuss two constructive expressions of the analogy between countably additive, positive linear functionals on $\mathscr{A}(H)$ and positive measures on the set of continuous, real-valued functions of compact support 
on a locally compact metric space. The work of $\S 3$ can be regarded as a first step towards a constructive theory of noncommutative integration [5].

We shall assume familiarity with those features which distinguish constructive mathematics from its classical (that is, traditional) counterpart, and with the foundations of constructive analysis described in [1], [2] and [3]. Throughout our paper, a result stated without proof should be regarded as an invitation to a little extra effort on the part of the reader.

1. Let $H$ be a separable, complex Hilbert space with scalar product $\langle\rangle,,\left(a_{n}\right)_{n \geq 1}$ an orthonormal basis of $H$, and $\mathscr{L}(H)$ the linear space of bounded operators on $H$. Define the strong operator norm \|\|$_{s}$ and the weak operator norm \|\|$_{w}$ on $\mathscr{L}(H)$ by setting

$$
\begin{aligned}
\|T\|_{s} & =\sum_{n=1}^{\infty} 2^{-n}\left\|T a_{n}\right\|, \\
\|T\|_{w} & =\sum_{m, n=1}^{\infty} 2^{-m-n}\left|\left\langle T a_{m}, a_{n}\right\rangle\right|
\end{aligned}
$$

for each $T$ in $\mathscr{L}(H)$. Strong operator norms defined in terms of different orthonormal bases of $H$ induce equivalent metrics on the unit ball $\mathscr{L}_{1}(H)=\{T \in \mathscr{L}(H): \forall x \in H \quad(\|T x\| \leqq\|x\|)\}$ of $\mathscr{L}(H)$; the same holds for weak operator norms. Note that $\mathscr{L}_{1}(H)$ is a closed, totally bounded subset of $\mathscr{L}(H)$ in the metric induced by the weak operator norm; but that the weak operator compactness of $\mathscr{L}_{1}(H)$ is an essentially nonconstructive proposition [4].

A constructive proof that every element of $\mathscr{L}(H)$ has an adjoint would comprise a finite routine which, applied to any bounded operator $T$ on $H$, constructs the adjoint of $T$. It seems unlikely that such a routine will ever be produced. However, if $\mathscr{A}(H)$ denotes the set of all elements of $\mathscr{L}(H)$ for which the adjoint is computable, an examination of the proof of the theorem in [4] shows that $\mathscr{L}_{1}(H)=\mathscr{A}(H) \cap \mathscr{L}_{1}(H)$ is weak operator norm dense in $\mathscr{L}_{1}(H)$. Moreover, if $H$ is finite dimensional, then $\mathscr{A}(H)=\mathscr{L}(H)$.

An element $A$ of $\mathscr{L}(H)$ is said to be positive if it is selfadjoint and $\langle A x, x\rangle \geqq 0$ for each $x$ in $H$. If $A, B$ are selfadjoint and $A-B$ is positive, we write $A \geqq B$. If $A \in \mathscr{A}(H)$, then $A^{*} A \geqq 0$; we write $|A|$ for the positive square root of $A^{*} A$. The existence of $|A|$ is a consequence of the functional calculus for $A^{*} A[3, \mathrm{Ch} .6]$.

An element $U$ of $\mathscr{A}(H)$ is said to be a partial isometry if there exists a projection $P$, called the initial projection of $U$, such that $\|U P x\|=\|P x\|$ and $U(I-P) x=0$ for each $x$ in $H$. (As usual, $I$ denotes the identity operator $x \rightarrow x$ on $H$.) An element $U$ of $\mathscr{A}(H)$ is a partial isometry if and only if $U^{*} U$ is a projection; in 
which case $U^{*} U$ is the initial projection of $U$, and $U^{*}$ is a partial isometry with initial projection $U U^{*}$.

Of considerable importance in classical operator theory is the polar decomposition of an operator $S: S=U|S|$, with $U$ a partial isometry. Such a polar decomposition is not always possible in constructive mathematics, as we may be unable to construct the projection on the closure of the range of $S$ [cf. 6, p. 15]. Fortunately, we can get by with the following constructive substitute.

Theorem 1.1. (Approximate Polar Decomposition). Let $A \in$ $\mathscr{A}(H)$, and let $\varepsilon>0$. Then there exists a partial isometry $U$ such that $\varepsilon$ is a bound for both $A-U|A|$ and $|A|-U^{*} A$.

Proof. Without loss of generality, we assume that $A \in \mathscr{A} \mathscr{A}_{1}(H)$. Let $\left(\mu, f \rightarrow f\left(A^{*} A\right)\right)$ be the functional calculus for $A^{*} A[3, \mathrm{Ch} .6, \S 6]$, and compute a strictly decreasing sequence $\left(\alpha_{n}\right)_{n \geqq 1}$ of positive numbers converging to 0 such that the complemented sets

$$
S(n)=\left(\left\{x \in X: \operatorname{pr}_{1}(x) \leqq-\alpha\right\}, \quad\left\{x \in X: \operatorname{pr}_{1}(x)>-\alpha\right\}\right)
$$

are all integrable [3, Ch. 5, 6.5]. (Here, $\operatorname{pr}_{1}$ is the mapping $\left(x_{n}\right)_{n \geqq 1} \rightarrow$ $x_{1}$ on the compact product metric space $X=\prod_{n=1}^{\infty}[-1,1]$ underlying the integral $\mu$.) Were $\mu S(n)>0$, it would follow from the inequality $\left(\left|\mathrm{pr}_{1}\right|-\mathrm{pr}_{1}-2 \alpha_{n}\right) \chi_{S(n)} \geqq 0$ and [3, Ch. 6, 6.3] that there exists $x \in H$ with $\|x\|=1$ and $\left\langle\left(\left|A^{*} A\right|-A^{*} A\right) x, x\right\rangle \geqq 2 \alpha_{n}>0$. As this contradicts the fact that $\left|A^{*} A\right|=A^{*} A$, we conclude that $\mu S(n)=0$. It follows that the complemented set $\left(\mathrm{pr}_{1}<0\right)$, with characteristic function $\sup _{n \geq 1} \chi_{S(n)}$, has measure zero. Thus, by [3, Ch. 5, 5.1], $F=\left\{x \in X: \operatorname{pr}_{1}(x) \geqq 0\right\}$ is a full set. As $\left|\mathrm{pr}_{1}\right|=\mathrm{pr}_{1}$ throughout $F$, we have

$$
A^{*} A=\left|A^{*} A\right|=\left|\operatorname{pr}_{1}\right|\left(A^{*} A\right)=\operatorname{pr}_{1}\left(A^{*} A\right) \text {. }
$$

Now choose $\alpha$ in $(0, \varepsilon)$ so that the complemented set

$$
S=\left(\operatorname{pr}_{1} \geqq \alpha\right)=\left(\left\{x \in X: \operatorname{pr}_{1}(x) \geqq \alpha\right\},\left\{x \in X: \operatorname{pr}_{1}(x)<\alpha\right\}\right)
$$

is integrable, and let $\chi=\chi_{s}, \phi=\left(\mathrm{pr}_{1} \vee \alpha\right)^{-1}\left(\mathrm{pr}_{1} \vee 0\right)^{1 / 2} \chi$ and $U=$ $A \dot{\phi}\left(A^{*} A\right)$. As the domain $G$ of $\chi$ is a full set and $\operatorname{pr}_{1} \dot{\phi}^{2}=\chi=\chi^{2}$ on $G$, we have

$$
\begin{aligned}
U^{*} U & =\dot{\phi}\left(A^{*} A\right) A^{*} A \dot{\varphi}\left(A^{*} A\right) \\
& =\dot{A}^{*} A \dot{\phi}\left(A^{*} A\right)^{2} \\
& =\left(\operatorname{pr}_{1} \phi^{2}\right)\left(A^{*} A\right) \\
& =\chi\left(A^{*} A\right),
\end{aligned}
$$


and $\chi\left(A^{*} A\right)=\chi\left(A^{*} A\right)^{2}$. (Note that $A^{*} A$ commutes with every operator $f\left(A^{*} A\right)$ defined by the functional calculus for $A^{*} A$.) Hence $\chi\left(A^{*} A\right)$ is a projection, and $U$ is a partial isometry.

Throughout the full set $F \cap G$, we have $\left|\operatorname{pr}_{1}\left(1-\phi \mathrm{pr}_{1}^{1 / 2}\right)\right| \leqq \alpha$ and $\operatorname{pr}_{1}^{1 / 2} \phi=\chi$. Hence, by [3, Ch. 6, 6.1], $\alpha$ is a bound for $A^{*} A(I-$ $\left.\phi\left(A^{*} A\right)|A|\right)$, and $|A| \phi\left(A^{*} A\right)=\chi\left(A^{*} A\right)$. For each $x \in H$ with $\|x\| \leqq$ 1 , we therefore have

$$
\begin{aligned}
& \|(A-U|A|) x\|^{2} \\
& \quad=\left\langle\left(A^{*}-|A| U^{*}\right)(A-U|A|) x, x\right\rangle \\
& \quad \leqq\left|\left\langle A^{*} A\left(I-\phi\left(A^{*} A\right)|A|\right) x, x\right\rangle\right|+\left|\left\langle\left(|A| \dot{\phi}\left(A^{*} A\right) A^{*} A-U^{*} U|A|^{2}\right) x, x\right\rangle\right| \\
& \quad \leqq \alpha+\left|\left\langle\left(|A| \phi\left(A^{*} A\right)-\chi\left(A^{*} A\right)\right) A^{*} A x, x\right\rangle\right| \\
& \quad<\varepsilon .
\end{aligned}
$$

Thus $\varepsilon$ is a bound for $A-U|A|$. Similar considerations, the details of which we omit, enable us to show that $\varepsilon$ is a bound for $|A|$ $U^{*} A$.

An element $A$ of $\mathscr{A}(H)$ is said to be a Hilbert-Schmidt operator if $\sum_{n=1}^{\infty}\left\|A a_{n}\right\|^{2}$ converges; in which case the sum of this series is independent of the orthonormal basis $\left(a_{n}\right)$, and we write $\|A\|_{2}$ for $\left(\sum_{n=1}^{\infty}\left\|A a_{n}\right\|^{2}\right)^{1 / 2}$. If $A$ is a Hilbert-Schmidt operator, then so is $A^{*}$; if also $B \in \mathscr{A}(H)$ and $\beta$ is a bound for $B$, then $A B$ and $B A$ are Hilbert-Schmidt operators, $\|A B\|_{2} \leqq \beta\|A\|_{2}$ and $\|B A\|_{2} \leqq \beta\|A\|_{2}$.

An operator $A \in \mathscr{A}(H)$ is said to be of trace class if $\|A\|_{1}=$ $\sum_{n=1}^{\infty}\left\langle|A| a_{n}, a_{n}\right\rangle$ converges. In that case, $\|A\|_{1}=\sum_{n=1}^{\infty}\left\||A|^{1 / 2} a_{n}\right\|^{2}$; so that $|A|^{1 / 2}$ is a Hilbert-Schmidt operator and $\|A\|_{1}$ is independent of the orthonormal basis $\left(a_{n}\right)$. Linear combinations of trace class operators are also of trace class.

Before discussing trace class operators any further, it is convenient to state the following simple consequence of the CauchySchwarz inequalities in $H$ and $\boldsymbol{C}^{n}$.

Lemma 1.2. Let $A$ be a positive operator, $R$ and $S$ elements of $\mathscr{L}(H)$, and $p, q$ positive integers with $q>p$. Then

$$
\begin{aligned}
\sum_{p+1}^{q} \mid & \left\langle A^{1 / 2} R a_{n}, A^{1 / 2} S a_{n}\right\rangle \mid \\
& \leqq\left(\sum_{p+1}^{q}\left\|A^{1 / 2} R a_{n}\right\|^{2}\right)^{1 / 2}\left(\sum_{p+1}^{q}\left\|A^{1 / 2} S a_{n}\right\|^{2}\right)^{1 / 2} .
\end{aligned}
$$

Let $A$ be of trace class, let $B \in \mathscr{A}(H)$, and let $\beta$ be a bound for $B$. Given $\varepsilon>0$, construct partial isometries $U, V$ so that $\varepsilon$ is a bound for both $|A B|-U^{*} A B$ and $A-V|A|$. For $q>p$, we have 


$$
\begin{aligned}
& \sum_{p+1}^{q}\left\langle|A B| a_{n}, a_{n}\right\rangle \\
& \leqq \sum_{p+1}^{q}\left|\left\langle\left(|A B|-U^{*} A B\right) a_{n}, a_{n}\right\rangle\right|+\sum_{p+1}^{q}\left|\left\langle U^{*}(A-V|A|) B a_{n}, a_{n}\right\rangle\right| \\
& +\sum_{p+1}^{q}\left|\left\langle U^{*} V|A| B a_{n}, a_{n}\right\rangle\right| \\
& \leqq \varepsilon(1+\beta)(q-p)+\sum_{p+1}^{q}\left|\left\langle|A|^{1 / 2} B a_{n},|A|^{1 / 2} V^{*} U a_{n}\right\rangle\right| \\
& \leqq \varepsilon(1+\beta)(q-p)+\left(\sum_{n=p+1}^{q}\left\||A|^{1 / 2} B a_{n}\right\|^{2}\right)^{1 / 2}\left(\sum_{n=p+1}^{q}\left\||A|^{1 / 2} V^{*} U a_{n}\right\|^{2}\right)^{1 / 2} \\
& \leqq \varepsilon(1+\beta)(q-p)+\left(\sum_{p+1}^{q}\left\||A|^{1 / 2} B a_{n}\right\|^{2}\right)^{1 / 2}\left\||A|^{1 / 2} V^{*} U\right\|_{2} .
\end{aligned}
$$

As $\left\||A|^{1 / 2} V^{*} U\right\|_{2} \leqq\left\||A|^{1 / 2}\right\|_{2}=\|A\|_{1}^{1 / 2}$, and as $\varepsilon>0$ is arbitrary, it follows that

$$
\sum_{p+1}^{q}\left\langle|A B| a_{n}, a_{n}\right\rangle \leqq\|A\|_{1}^{1 / 2}\left(\sum_{p+1}^{q}\left\||A|^{1 / 2} B a_{n}\right\|^{2}\right)^{1 / 2} \quad(q>p) .
$$

As $|A|^{1 / 2} B$ is a Hilbert-Schmidt operator, we see that $\sum_{1}^{\infty}\left\langle|A B| a_{n}, a_{n}\right\rangle$ converges, and hence that $A B$ is of trace class. Moreover, $\|A B\|_{1} \leqq$ $\|A\|_{1}^{1 / 2}\left\||A|^{1 / 2} B\right\|_{2} \leqq \beta\|A\|_{1}$. A similar argument using approximate polar decompositions shows that

$$
\sum_{p+1}^{q}\left\langle|B A| a_{n}, a_{n}\right\rangle \leqq \beta\|A\|_{1}^{1 / 2}\left(\sum_{p+1}^{q}\left\||A|^{1 / 2} a_{n}\right\|^{2}\right)^{1 / 2} \quad(q>p) .
$$

Hence $B A$ is of trace class, and $\|B A\|_{1} \leqq \beta\|A\|_{1}$.

Taking $B=I$ in (1.3), we obtain

$$
\sum_{p+1}^{q}\left|\left\langle A a_{n}, a_{n}\right\rangle\right| \leqq \sum_{p+1}^{q}\left\langle|A| a_{n}, a_{n}\right\rangle \leqq\|A\|_{1}^{1 / 2}\left(\sum_{p+1}^{q}\left\langle|A| a_{n}, a_{n}\right\rangle\right)^{1 / 2}
$$

It follows that $\sum_{1}^{\infty}\left\langle A a_{n}, a_{n}\right\rangle$ converges absolutely and that $\sum_{n=1}^{\infty}$ $\left|\left\langle A a_{n}, a_{n}\right\rangle\right| \leqq\|A\|_{1}$. Writing $A=(|A|+A) / 2-(|A|-A) / 2$, a linear combination of positive operators of trace class, we see that

$$
\operatorname{Tr}(A)=\sum_{n=1}^{\infty}\left\langle A a_{n}, a_{n}\right\rangle,
$$

the trace of $A$, is independent of the orthonormal basis $\left(a_{n}\right)$. If $A$ is of trace class and $B \in \mathscr{A}(H)$, then $\operatorname{Tr}(A B)=\operatorname{Tr}(B A)$ [6, p. 37].

If $A, B$ are operators of Hilbert-Schmidt type, then an argument using the approximate polar decompositions of $A B$ and $A$ shows that

$$
\sum_{p+1}^{q}\left\langle|A B| a_{n}, a_{n}\right\rangle \leqq\left(\sum_{1}^{\infty}\left\|A^{*} a_{n}\right\|^{2}\right)^{1 / 2}\left(\sum_{p+1}^{q}\left\|B a_{n}\right\|^{2}\right)^{1 / 2} \quad(q>p) ;
$$


whence $A B$ is of trace class. Similarly, $B A$ is of trace class. Writing $B a_{n}=\sum_{k=1}^{\infty}\left\langle B a_{n}, a_{k}\right\rangle a_{k}$, we easily prove that $\operatorname{Tr}(A B)=\operatorname{Tr}(B A)$.

If $A$ is of trace class, then $B \rightarrow \operatorname{Tr}(A B)=\operatorname{Tr}(B A)$ is a linear mapping of $\mathscr{A}(H)$ into $C$. This mapping is uniformly continuous on $\mathscr{A}_{1}(H)$ with respect to the weak operator norm, and so extends to a linear functional $\phi_{A}$ on $\mathscr{L}(H)$ that is weak operator uniformly continuous on $\mathscr{L}_{1}(H)$. As $\mathscr{L}_{1}(H)$ is weak operator totally bounded, $\dot{\phi}_{A}$ is normable: that is, $\left\|\dot{\phi}_{A}\right\|=\sup \left\{\left|\dot{\phi}_{A}(B)\right|: B \in \mathscr{L}_{1}(H)\right\}$ is computable.

For each $B$ in $\mathscr{A}_{1}(H)$,

$$
\left|\phi_{A}(B)\right| \leqq \sum_{1}^{\infty}\left|\left\langle A B a_{n}, a_{n}\right\rangle\right| \leqq\|A B\|_{1} \leqq\|A\|_{1} .
$$

On the other hand, given $\varepsilon$ in $(0,1)$, and computing in turn a positive integer $\nu$ and a partial isometry $U$ such that $\sum_{v+1}^{\infty}\left\langle|A| a_{n}, a_{n}\right\rangle<\varepsilon^{2}$ and $\varepsilon \nu^{-1}$ is a bound for $|A|-U^{*} A$, we have (by (1.4))

$$
\begin{aligned}
& \left|\phi_{A}\left(U^{*}\right)\right|=\left|\sum_{n=1}^{\infty}\left\langle U^{*} A a_{n}, a_{n}\right\rangle\right| \\
& \geqq\|A\|_{1}-\sum_{n=1}^{\nu}\left|\left\langle\left(|A|-U^{*} A\right) a_{n}, a_{n}\right\rangle\right| \\
& \quad-\sum_{n=\nu+1}^{\infty}\left\langle|A| a_{n}, a_{n}\right\rangle-\sum_{n=\nu+1}^{\infty}\left|\left\langle U^{*} A a_{n}, a_{n}\right\rangle\right| \\
& \geqq\|A\|_{1}-\varepsilon-\varepsilon^{2}-\|A\|_{1}^{1 / 2}\left(\sum_{\nu+1}^{\infty}\left\||A|^{1 / 2} a_{n}\right\|^{2}\right)^{1 / 2} \\
& \geqq\|A\|_{1}-2 \varepsilon-\|A\|_{1}^{1 / 2}\left(\sum_{\nu+1}^{\infty}\left\langle|A| a_{n}, a_{n}\right\rangle\right)^{1 / 2} \\
& \geqq\|A\|_{1}-\varepsilon\left(2+\|A\|_{1}^{1 / 2}\right) .
\end{aligned}
$$

It now follows that $\left\|\phi_{A}\right\|=\|A\|_{1}$.

The set of trace class operators on $H$, taken with the trace class norm \|\|$_{1}$, is a normed linear space over $C$. We prove that this space is \|\|$_{1}$-complete. Let $\left(A_{k}\right)_{k \geq 1}$ be a \|\|$_{1}$-Cauchy sequence of trace class operators. As $\left\|A_{j}-A_{k}\right\|_{1}$ is a bound for $A_{j}-A_{k}$, $\left(A_{k}\right)_{k \geq 1}$ converges in bound (that is, uniformly on the unit ball of $H$ ) to an element $A$ of $\mathscr{L}(H)$; as $\left\|A_{j}-A_{k}\right\|_{1}$ is also a bound for $A_{j}^{*}-A_{k}^{*},\left(A_{k}^{*}\right)_{k \geqq 1}$ converges in bound to an element of $\mathscr{L}(H)$ which is clearly the adjoint of $A$. Given $\varepsilon>0$, compute $k(\varepsilon)$ so that $\left\|A_{j}-A_{k}\right\|_{1} \leqq \varepsilon$ whenever $j, k \geqq k(\varepsilon)$. If $U$ is a partial isometry and $q>p$, we have

$$
\sum_{p+1}^{q}\left|\left\langle U^{*} A_{k} a_{n}, a_{n}\right\rangle\right| \leqq\left\|A_{k}-A_{k(\varepsilon)}\right\|_{1}+\sum_{p+1}^{q}\left|\left\langle U^{*} A_{k(\varepsilon)} a_{n}, a_{n}\right\rangle\right|
$$

for each $k \geqq 1$, and therefore

$$
\sum_{p+1}^{q}\left\langle|A| a_{n}, a_{n}\right\rangle
$$




$$
\begin{aligned}
& \leqq \sum_{p+1}^{q}\left|\left(\left\langle|A|-U^{*} A\right) a_{n}, a_{n}\right\rangle\right|+\lim _{k \rightarrow \infty} \sum_{p+1}^{q}\left|\left\langle U^{*} A_{k} a_{n}, a_{n}\right\rangle\right| \\
& \leqq \sum_{p+1}^{q}\left|\left\langle\left(|A|-U^{*} A\right) a_{n}, a_{n}\right\rangle\right|+\varepsilon+\sum_{p+1}^{q}\left|\left\langle U^{*} A_{k(\varepsilon)} a_{n}, a_{n}\right\rangle\right| \\
& \leqq \sum_{p+1}^{q}\left|\left\langle\left(|A|-U^{*} A\right) a_{n}, a_{n}\right\rangle\right|+\varepsilon+\sum_{p+1}^{q}\left\langle\left|U^{*} A_{k(\varepsilon)}\right| a_{n}, a_{n}\right\rangle \\
& \leqq \sum_{p+1}^{q}\left|\left\langle\left(|A|-U^{*} A\right) a_{n}, a_{n}\right\rangle\right|+\varepsilon+\left\|A_{k(\varepsilon)} \mid\right\|_{1}^{1 / 2}\left(\sum_{p+1}^{q}\left\|\left|A_{k(\varepsilon)}\right|^{1 / 2} a_{n}\right\|^{2}\right)^{1 / 2}
\end{aligned}
$$

the last step following from (1.4). In view of (1.1), we have

$$
\sum_{p+1}^{q}\left\langle|A| a_{n}, a_{n}\right\rangle \leqq \varepsilon+\left\|A_{k(\varepsilon)}\right\|_{1}^{1 / 2}\left(\sum_{p+1}^{q}\left\|\left|A_{k(\varepsilon)}\right|^{1 / 2} a_{n}\right\|^{2}\right)^{1 / 2} \quad(q>p) .
$$

It follows that $A$ is of trace class. On the other hand, if $\nu \geqq 1$ and $k \geqq k(\varepsilon)$, we have

$$
\sum_{1}^{\nu}\left|\left\langle U^{*}\left(A_{j}-A_{k}\right) a_{n}, a_{n}\right\rangle\right| \leqq\left\|A_{j}-A_{k}\right\|_{1} \leqq \varepsilon \quad(j \geqq k(\varepsilon)),
$$

and therefore

$$
\begin{aligned}
& \sum_{1}^{\nu}\left\langle\left|A-A_{k}\right| a_{n}, a_{n}\right\rangle \\
& \leqq \sum_{1}^{\nu}\left|\left\langle\left(\left|A-A_{k}\right|-U^{*}\left(A-A_{k}\right)\right) a_{n}, a_{n}\right\rangle\right| \\
& +\lim _{j \rightarrow \infty} \sum_{1}^{\nu}\left|\left\langle U^{*}\left(A_{j}-A_{k}\right) a_{n}, a_{n}\right\rangle\right| \\
& \leqq \sum_{1}^{\nu}\left|\left\langle\left(\left|A-A_{k}\right|-U^{*}\left(A-A_{k}\right)\right) a_{n}, a_{n}\right\rangle\right|+\varepsilon .
\end{aligned}
$$

It follows from (1.1) that $\sum_{1}^{\nu}\left\langle\left|A-A_{k}\right| a_{n}, a_{n}\right\rangle \leqq \varepsilon$ whenever $k \geqq k(\varepsilon)$ and $\nu \geqq 1$, and therefore that $\lim _{k \rightarrow \infty}\left\|A-A_{k}\right\|_{1}=0$.

2. Let $\mathscr{R}$ be a linear subset of $\mathscr{L}(H)$, and $\phi$ a linear mapping of $\mathscr{R}$ into $C$. We say that $\phi$ is ultraweakly (resp. ultrastrongly) continuous on $\mathscr{R}$ if $\phi$ is uniformly continuous on $\mathscr{R} \cap \mathscr{L}_{1}(H)$ with respect to the weak (resp. strong) operator norm. If $\phi$ is ultraweakly continuous and $\mathscr{A}(H) \subset \mathscr{R}$, then $\phi$ extends to an ultraweakly continuous linear functional on $\mathscr{L}(H)$, and this extended functional is normable.

Classically, our condition of ultraweak continuity is equivalent to the pointwise continuity of $\phi$ with respect to the weak operator topology on $\mathscr{R}$. As uniform, rather than pointwise, continuity seems to be necessary for successful computation, and as there is no known constructive proof of the Uniform Continuity Theorem, we are obliged to build uniform continuity on $\mathscr{R} \cap \mathscr{L}_{1}(H)$ into our definition. 
We say that a linear mapping $\phi: \mathscr{R} \rightarrow C$ is positive, and write $\phi \geqq 0$, if $\phi(A) \geqq 0$ for each positive $A$ in $\mathscr{R}$. We then have $\phi\left(A^{*}\right)=$ $\dot{\phi}(A)^{*}$ for each $A$ in $\mathscr{R} \cap \mathscr{A}(H)$. If $\phi$ is positive and $A, B$ belong to $\mathscr{R} \cap \mathscr{A}(H)$, we have the following Cauchy-Schwarz inequality: $\left|\phi\left(A^{*} B\right)\right|^{2} \leqq \phi\left(A^{*} A\right) \phi\left(B^{*} B\right)$. Moreover, if $\mathscr{A}(H) \subset \mathscr{R}$ then $\phi$ is also bounded; in fact, it is normable, and $\|\phi\|=\phi(I)$.

If $I \in \mathscr{R}$, and $\phi(I)=\sum_{n=1}^{\infty} \phi\left(P_{n}\right)$ whenever $\left(P_{n}\right)_{n \geqq 1}$ is a sequence of pairwise orthogonal projections in $\mathscr{R}$ whose sum in the strong operator norm is $I$, then we say that $\phi$ is countably additive.

We now give constructive proofs of certain relations between the various types of linear mapping defined above.

THEOREM 2.1. The following are equivalent conditions on a positive linear functional $\phi$ on $\mathscr{S}(H)$ :

(i) $\phi$ is countably additive.

(ii) $\dot{\phi}$ is ultrastrongly continuous.

(iii) $\dot{\phi}$ is ultraweakly continuous.

(iv) there exists a positive operator $A$ of trace class such that $\phi(S)=\operatorname{Tr}(S A)$ for each $S$ in $\mathscr{A}(H)$.

( v) there exists a sequence $\left(x_{n}\right)_{n \geqq 1}$ in $H$ such that $\sum_{n=1}^{\infty}\left\|x_{n}\right\|^{2}$ converges, and $\phi(S)=\sum_{n=1}^{\infty}\left\langle S x_{n}, x_{n}\right\rangle$ for each $S$ in $\mathscr{A}(H)$.

Proof. Although several parts of the following proof are based on fairly well-known classical arguments, we shall give most of the details of the proof, for the sake of completeness. For each $n \geqq 1$, let $P_{n}$ be the projection of $H$ on $C a_{n}$ and let $Q_{n}=\sum_{k=1}^{n} P_{k}$. Then $Q_{n}$ is the projection of $H$ on the span of $\left\{a_{1}, \cdots, a_{n}\right\}$. Define also elements $S_{j, k}$ of $\mathscr{A}(H)$ by setting $S_{j, k} x=\left\langle x, a_{j}\right\rangle a_{k}$ whenever $x \in H$, $j \geqq 1$ and $k \geqq 1$. Note that, if $A \in \mathscr{L}(H)$ and $n \geqq 1$, then $P_{n} A P_{n}=$ $\left\langle A a_{n}, a_{n}\right\rangle P_{n}$ and $Q_{n} A Q_{n}=\sum_{j, k=1}^{n}\left\langle A a_{j}, a_{k}\right\rangle S_{j, k}$.

(i ) $\Rightarrow$ (ii) Suppose that $\phi$ is countably additive. Define linear mappings $\phi_{n}: \mathscr{A}(H) \rightarrow C$ by setting $\phi_{n}(S)=\sum_{k=1}^{n} \phi\left(S P_{k}\right)$ for each $S$ in $\mathscr{A}(H)$ and each $n \geqq 1$. Given $S$ in $\mathscr{A}(H)$ and using the CauchySchwarz inequality for positive linear functionals, we have

$$
\begin{aligned}
\left|\phi_{n}(S)\right| & \leqq \sum_{k=1}^{n} \phi(I)^{1 / 2} \phi\left(P_{k} S^{*} S P_{k}\right)^{1 / 2} \\
& =\phi(I)^{1 / 2} \sum_{k=1}^{n} \phi\left(P_{k}\right)^{1 / 2}\left\langle S^{*} S a_{k}, a_{k}\right\rangle^{1 / 2} \\
& \leqq \phi(I)^{1 / 2} \sum_{k=1}^{n} \phi(I)^{1 / 2}\left\|S a_{k}\right\| \\
& =\phi(I) \sum_{k=1}^{n}\left\|S a_{k}\right\|
\end{aligned}
$$


It readily follows from this that $\phi_{n}$ is ultrastrongly continuous. Moreover, for $S \in \mathscr{\mathscr { A } _ { 1 } ( H )}$

$$
\begin{aligned}
\left|\phi(S)-\dot{\phi}_{n}(S)\right| & =\left|\dot{\phi}\left(S\left(I-\sum_{k=1}^{n} P_{k}\right)\right)\right| \\
& \leqq \phi\left(S S^{*}\right)^{1 / 2}\left(\phi(I)-\sum_{k=1}^{n} \phi\left(P_{k}\right)\right)^{1 / 2} \\
& \leqq \phi(I)^{1 / 2}\left(\phi(I)-\sum_{k=1}^{n} \phi\left(P_{k}\right)\right)^{1 / 2} .
\end{aligned}
$$

As $\phi$ is countably additive, it follows that $\phi_{n}(S)$ converges to $\phi(S)$ uniformly on $\mathscr{A}_{1}(H)$; whence $\phi$ is ultrastrongly continuous.

(ii) $\Rightarrow$ (iii) Suppose $\phi$ is ultrastrongly continuous. Let $\delta$ be a modulus of continuity for $\phi$ on $\left(\mathscr{A}_{1}(H),\|\|_{s}\right)$, and let $\varepsilon>0$. Compute $\nu \geqq 1$ so that $\dot{\phi}\left(I-Q_{\nu}\right) \leqq \varepsilon^{2} / 9(1+\phi(I))$ and $\sum_{k=\nu+1}^{\infty} 2^{-k} \leqq \delta(\varepsilon / 3)$. Given $S$ in $\mathscr{A}_{1}(H)$, we have $\left\|S\left(I-Q_{\nu}\right)\right\|_{s} \leqq \delta(\varepsilon / 3)$, and therefore $\left|\phi\left(S\left(I-Q_{\nu}\right)\right)\right| \leqq \varepsilon / 3$. Also,

$$
\begin{aligned}
\left|\dot{\phi}\left(\left(I-Q_{\nu}\right) S Q_{\nu}\right)\right|^{2} & \leqq \dot{\phi}\left(I-Q_{\nu}\right) \dot{\phi}\left(Q_{\nu} S^{*} S Q_{\nu}\right) \\
& \leqq \phi\left(I-Q_{\nu}\right) \dot{\phi}(I) \\
& \leqq \varepsilon^{2} / 9,
\end{aligned}
$$

and so $\left|\phi\left(\left(I-Q_{\nu}\right) S Q_{\nu}\right)\right| \leqq \varepsilon / 3$. Finally, as all norms on the finitedimensional space $\mathscr{L}\left(Q_{\nu}(H)\right)$ are equivalent, there exists $\kappa>0$ such that, for all $S$ in $\mathscr{L}(H)$,

$$
\left\|Q_{\nu} S Q_{\nu}\right\|_{s}=\sum_{k=1}^{\nu} 2^{-k}\left\|Q_{\nu} S a_{k}\right\| \leqq \kappa \sum_{j, k=1}^{\nu} 2^{-j-k}\left|\left\langle Q_{\nu} S a_{j}, a_{k}\right\rangle\right| \leqq \kappa\|S\|_{w} .
$$

Taken with the identity

$$
\phi(S)=\dot{\phi}\left(Q_{\nu} S Q_{\nu}\right)+\phi\left(S\left(I-Q_{\nu}\right)\right)+\phi\left(\left(I-Q_{\nu}\right) S Q_{\nu}\right),
$$

all this shows that $|\phi(S)| \leqq \varepsilon$ whenever $S \in \mathscr{L}_{1}(H)$ and $\|S\|_{w} \leqq \kappa^{-1} \delta(\varepsilon / 3)$; whence $\phi$ is ultraweakly continuous.

(iii) $\Rightarrow$ (iv) Suppose that $\phi$ is ultraweakly continuous. For each positive integer $n$, define $A(n)=\sum_{j, k=1}^{n} \phi\left(S_{k, j}\right) S_{j, k}$. Then $A(n)$ is a selfadjoint, positive operator of trace class. For each $S$ in $\mathscr{A}(H)$, $\phi\left(Q_{n} S Q_{n}\right)=\phi_{A(n)}(S)$, and so

$$
\left|\phi(S)-\phi_{A(n)}(S)\right| \leqq\left|\phi\left(S\left(I-Q_{n}\right)\right)\right|+\left|\phi\left(\left(I-Q_{n}\right) S Q_{n}\right)\right| .
$$

As \|\|$_{w} \leqq\|\|_{s}, \phi$ is ultrastrongly continuous, and an argument used above shows that $\left\|\phi-\dot{\phi}_{A(n)}\right\| \rightarrow 0$ as $n \rightarrow \infty$. As the linear functional $\phi-\phi_{A(j)}$ is ultraweakly continuous, it is normable. Moreover, 


$$
\begin{aligned}
\|A(j)-A(k)\|_{1} & =\left\|\phi_{A(j)-A(k)}\right\| \\
& =\left\|\phi_{A(j)}-\phi_{A(k)}\right\| \\
& \leqq\left\|\phi-\phi_{A(j)}\right\|+\left\|\phi-\phi_{A(k)}\right\|,
\end{aligned}
$$

and so $(A(k))_{k \geqq 1}$ is a \|\|$_{1}$-Cauchy sequence. Thus there exists a trace class operator $A$ such that $\lim _{k \rightarrow \infty}\|A-A(k)\|_{1}=0$. As $\|A-A(k)\|_{1}$ is a bound for $A-A(k),(A(k))_{k \geq 1}$ converges to $A$ in bound; so that $A$ is selfadjoint and positive. Finally, as

$$
\begin{aligned}
\left\|\phi-\phi_{A}\right\| & \leqq\left\|\phi-\phi_{A(k)}\right\|+\left\|\phi_{A-A(k)}\right\| \\
& =\left\|\phi-\phi_{A(k)}\right\|+\|A-A(k)\|_{1},
\end{aligned}
$$

we have $\left\|\dot{\phi}-\phi_{A}\right\|=0, \phi=\phi_{A}$, and therefore $\phi(S)=\operatorname{Tr}(S A)$ for each $S$ in $\mathscr{A}(H)$.

(iv) $\Rightarrow$ (v) Suppose that (iv) obtains, and let $S \in \mathscr{A}(H)$. Then $A^{1 / 2}$ and $S A^{1 / 2}$ are Hilbert-Schmidt operators, and so $A^{1 / 2} S A^{1 / 2}$ is of trace class. Moreover,

$$
\begin{aligned}
\phi(S) & =\operatorname{Tr}\left(S A^{1 / 2} A^{1 / 2}\right) \\
& =\operatorname{Tr}\left(A^{1 / 2} S A^{1 / 2}\right) \\
& =\sum_{n=1}^{\infty}\left\langle A^{1 / 2} S A^{1 / 2} a_{n}, a_{n}\right\rangle \\
& =\sum_{n=1}^{\infty}\left\langle S x_{n}, x_{n}\right\rangle,
\end{aligned}
$$

where $x_{n}=A^{1 / 2} a_{n}$ and $\sum_{n=1}^{\infty}\left\|x_{n}\right\|^{2}=\operatorname{Tr}(A)$ converges.

$(\mathrm{v}) \Rightarrow(\mathrm{i})$ We omit the comparatively trivial details of this part of the proof.

COROLLARY 2.2. If $\phi$ is a countably additive, positive linear functional on $\mathscr{L}(H)$, then $\phi$ is of the form $S \rightarrow \sum_{n=1}^{\infty}\left\langle S x_{n}, x_{n}\right\rangle$, where each $x_{n}$ belongs to $H$ and $\sum_{n=1}^{\infty}\left\|x_{n}\right\|^{2}$ converges.

In classical mathematics, the expression of a compact operator $A$ in diagonal form enables us to add the following to the list of equivalent conditions in (2.2):

(vi) there exists an orthonormal basis $\left(e_{n}\right)_{n \geq 1}$ of $H$, and a sequence $\left(\lambda_{n}\right)_{n \geqq 1}$ of nonnegative numbers, such that $\phi(S)=\sum_{n=1}^{\infty} \lambda_{n}\left\langle S e_{n}, e_{n}\right\rangle$ for each $S$ in $\mathscr{A}(H)$.

It is easy to see that, if (iv) implies (vi), then the trace class operator $A$ in (iv) can be expressed in diagonal form. The following argument shows that, even in a two-dimensional Hilbert space, this 
expression may not be possible by constructive means.

Let $x$ and $y$ be nonnegative numbers with $x y=0$ and $y<1$, and let $A$ be the operator on $C^{2}$ with matrix $\left[\begin{array}{cc}1+x & y \\ y & 1+y\end{array}\right]$. Then $A$ is a positive, compact operator on the Hilbert space $C^{2}$. Suppose that $A$ can be expressed in diagonal form; so that there exist complex numbers $\lambda, u, v$ such that $|u|^{2}+|v|^{2} \neq 0$ and $\left[\begin{array}{cc}1+x & y \\ y & 1+y\end{array}\right]\left[\begin{array}{l}u \\ v\end{array}\right]=$ $\lambda\left[\begin{array}{l}u \\ v\end{array}\right]$. Then $(1+x) u+y v=\lambda u, y u+(1+y) v=\lambda v,(1+x) u v+y v^{2}=$ $\lambda u v=y u^{2}+(1+y) u v$, and so

$$
x u v+y v^{2}=y u^{2}+y u v .
$$

Multiplying (*) through by $x$ we obtain $x^{2} u v=0$. On the other hand, multiplying (*) through by $y$, we obtain $y^{2}\left(u^{2}+u v-v^{2}\right)=0$. If $u \neq 0$, then we have either $v \neq 0$, in which case $x^{2}=0$ and so $x=0$; or $|v|<|u| / 2$. In the latter case, we have

$$
\left|u^{2}+u v-v^{2}\right| \geqq|u|^{2}-|u||v|-|v|^{2} \geqq|u|^{2} / 4,
$$

whence $y^{2}=0$, and therefore $y=0$. If $v \neq 0$, then, replacing $u$ by $v$ in this argument, we again find that either $x=0$ or $y=0$. It follows from all this that if we have constructive means of expressing any positive operator on the Hilbert space $C^{2}$ in diagonal form, then we have a constructive proof of the essentially nonconstructive proposition

$$
\forall x \geqq 0 \quad \forall y \geqq 0 \quad(x y=0 \Longrightarrow(x=0 \quad \text { or } \quad y=0)) .
$$

We conclude that a constructive proof that (iv) implies (vi) is unlikely to materialize.

3. In classical mathematics, there is a well-known analogy between countably additive, positive linear functionals on $\mathscr{L}(H)$ and positive integrals on $C(X)$, where $X$ is a locally compact metric space. In this analogy, projections correspond to characteristic functions of integrable sets, positive operators to nonnegative integrable functions, and expressions of the form $\langle A x, x\rangle$ to values of integrable functions.

In the constructive integration theory of Bishop and Cheng ([2], $[4$, Ch. 5]), countable additivity of an integral $\mu$ is replaced by the stronger property:

(†) If $f_{0}$ is an integrable function, and $\left(f_{n}\right)_{n \geqq 1}$ is a sequence of nonnegative integrable functions such that $\sum_{n=1}^{\infty} \mu\left(f_{n}\right)$ is convergent and less than $\mu\left(f_{0}\right)$, then there exists $x$ such that $\sum_{n=1}^{\infty} f_{n}(x)$ is convergent and less than $f_{0}(x)$. 
It is very easy to obtain the corresponding property of countably additive, positive linear functionals:

Proposition 3.1. Let $\phi$ be a countably additive, positive linear functional on $\mathscr{L}(H), A_{0}$ an element of $\mathscr{L}(H)$, and $\left(A_{n}\right)_{n \geqq 1}$ a sequence of positive, selfadjoint elements of $\mathscr{L}(H)$ such that $\sum_{n=1}^{\infty} \phi\left(A_{n}\right)$ is convergent and less than $\phi\left(A_{0}\right)$. Then there exists a unit vector $x$ in $H$ such that $\sum_{n=1}^{\infty}\left\langle A_{n} x, x\right\rangle$ is convergent and less than $\left\langle A_{0} x, x\right\rangle$.

Proof. By 2.2, $\phi$ is of the form $S \rightarrow \sum_{k=1}^{\infty}\left\langle S x_{k}, x_{k}\right\rangle$, where each $x_{k}$ belongs to $H$ and $\sum_{k=1}^{\infty}\left\|x_{k}\right\|^{2}$ converges. Thus $\sum_{n=1}^{\infty} \sum_{k=1}^{\infty}\left\langle A_{n} x_{k}, x_{k}\right\rangle$ converges to $\sum_{n=1}^{\infty} \phi\left(A_{n}\right)$. As each term $\left\langle A_{n} x_{k}, x_{k}\right\rangle$ is nonnegative for $n \geqq 1$, it follows that $\sum_{n=1}^{\infty}\left\langle A_{n} x_{k}, x_{k}\right\rangle$ converges for each $k$, and that $\sum_{k=1}^{\infty} \sum_{n=1}^{\infty}\left\langle A_{n} x_{k}, x_{k}\right\rangle$ converges to $\sum_{n=1}^{\infty} \phi\left(A_{n}\right)$. Hence

$$
\sum_{k=1}^{\infty}\left(\left\langle A_{0} x_{k}, x_{k}\right\rangle-\sum_{n=1}^{\infty}\left\langle A_{n} x_{k}, x_{k}\right\rangle\right)=\phi\left(A_{0}\right)-\sum_{n=1}^{\infty} \phi\left(A_{n}\right)>0,
$$

and so there exists $k$ with $\sum_{n=1}^{\infty}\left\langle A_{n} x_{k}, x_{k}\right\rangle\left\langle\left\langle A_{0} x_{k}, x_{k}\right\rangle\right.$. It only remains to set $x=\left\|x_{k}\right\|^{-1} x_{k}$.

In the special case where each $f_{n}$ is the characteristic function of an integrable set, the Bishop-Cheng property $(\dagger)$ of the integral $\mu$ enables us to construct $x$ such that $f_{0}(x)=1$, and $f_{n}(x)=0$ for each $n \geqq 1$. Our proof of the analogue of this property for countably additive, positive linear functionals requires the following lemma.

Lemma 3.2. If $P_{1}, \cdots, P_{n}$ are pairwise commuting projections on $H$, then $I-\prod_{k=1}^{n}\left(I-P_{k}\right) \leqq \sum_{k=1}^{n} P_{k}$.

Proof. For $n=1$ this is trivial. To complete an inductive proof, we observe that if $I-\prod_{k=1}^{m}\left(I-P_{k}\right) \leqq \sum_{k=1}^{m} P_{k}$ for some positive integer $m$, and if $x \in H$, then

$$
\begin{aligned}
\langle(I & \left.\left.-\prod_{k=1}^{m+1}\left(I-P_{k}\right)\right) x, x\right\rangle \\
& =\left\langle\left(I-\prod_{k=1}^{m}\left(I-P_{k}\right)\right) x, x\right\rangle+\left\langle P_{m+1}\left(\prod_{k=1}^{m}\left(I-P_{k}\right)\right) x, x\right\rangle \\
& \leqq\left\langle\sum_{k=1}^{m} P_{k} x, x\right\rangle+\left\|\left(\prod_{k=1}^{m}\left(I-P_{k}\right)\right) P_{m+1} x\right\|^{2} \\
& \leqq\left\langle\sum_{k=1}^{m} P_{k} x, x\right\rangle+\left\|P_{m+1} x\right\|^{2} \\
& =\left\langle\sum_{k=1}^{m} P_{k} x, x\right\rangle+\left\langle P_{m+1} x, x\right\rangle \\
& =\left\langle\sum_{k=1}^{m+1} P_{k} x, x\right\rangle .
\end{aligned}
$$


THEOREM 3.3. Let $\phi$ be a countably additive, positive linear functional on $\mathscr{L}(H)$ and $\left(P_{n}\right)_{n \geq 0}$ and sequence of pairwise commuting projections on $H$ such that $\sum_{n=1}^{\infty} \phi\left(P_{n}\right)$ is convergent and less then $\phi\left(P_{0}\right)$. Then there exists a unit vector $x$ in $H$ such that $P_{0} x=x$, and $P_{n} x=0$ for each $n \geqq 1$.

Proof. We first consider the special case where there exists $\xi$ in $H$ such that $\phi(R)=\langle R \xi, \xi\rangle$ for each $R$ in $\mathscr{L}(H)$. If $m, n$ are integers with $m>n \geqq 1$, then

$$
\begin{aligned}
\|\left(\prod_{k=1}^{m}\right. & \left.\left(I-P_{k}\right)\right) \xi-\left(\prod_{k=1}^{n}\left(I-P_{k}\right)\right) \xi \|^{2} \\
& =\left\|\left(\prod_{j=1}^{n}\left(I-P_{j}\right)\right)\left(I-\prod_{k=n+1}^{m}\left(I-P_{k}\right)\right) \xi\right\|^{2} \\
& \leqq\left\|\left(I-\prod_{k=n+1}^{m}\left(I-P_{k}\right)\right) \xi\right\|^{2} \\
& =\left\langle\left(I-\prod_{k=n+1}^{m}\left(I-P_{k}\right)\right) \xi, \xi\right\rangle \\
& \leqq\left\langle\sum_{k=n+1}^{m} P_{k} \xi, \xi\right\rangle \\
& =\sum_{k=n+1}^{m}\left\langle P_{k} \xi, \xi\right\rangle
\end{aligned}
$$

the second last step following from 3.1. As $\sum_{k=1}^{\infty}\left\langle P_{k} \xi, \xi\right\rangle=\sum_{k=1}^{\infty} \phi\left(P_{k}\right)$ is convergent, we see that $\left(\left(\prod_{k=1}^{n}\left(I-P_{k}\right)\right) \xi\right)_{n \geq 1}$ is a Cauchy sequence in $H$; whence we can compute

$$
\eta \equiv\left(\prod_{k=1}^{\infty}\left(I-P_{k}\right)\right) \xi=\lim _{n \rightarrow \infty}\left(\prod_{k=1}^{n}\left(I-P_{k}\right)\right) \xi .
$$

For each $n \geqq 1$, we clearly have $P_{n} P_{0} \eta=P_{0} P_{n} \eta=0$. On the other hand, writing

$$
\alpha \equiv\left(\left\|P_{0} \xi\right\|+\left\|\left(I-\prod_{k=1}^{n}\left(I-P_{k}\right)\right) \xi\right\|\right)^{-1},
$$

we have

$$
\begin{aligned}
& \left\langle\left(\prod_{k=1}^{n}\left(I-P_{k}\right)\right) \xi, P_{0} \xi\right\rangle \\
& \quad=\left\langle\xi, P_{0} \xi\right\rangle-\left\langle\left(I-\prod_{k=1}^{n}\left(I-P_{k}\right)\right) \xi, P_{0} \xi\right\rangle \\
& \geqq\left\|P_{0} \xi\right\|^{2}-\left\|\left(I-\prod_{k=1}^{n}\left(I-P_{k}\right)\right) \xi\right\|\left\|P_{0} \xi\right\| \\
& \quad=\left\|P_{0} \xi\right\|\left(\left\|P_{0} \xi\right\|-\left\|\left(I-\prod_{k=1}^{n}\left(I-P_{k}\right)\right) \xi\right\|\right) \\
& \quad=\left\|P_{0} \xi\right\| \alpha\left(\left\|P_{0} \xi\right\|^{2}-\left\|\left(I-\prod_{k=1}^{n}\left(I-P_{k}\right)\right) \xi\right\|^{2}\right)
\end{aligned}
$$




$$
\begin{aligned}
& =\left\|P_{0} \xi\right\| \alpha\left(\left\langle P_{0} \xi, \xi\right\rangle-\left\langle\left(I-\prod_{k=1}^{n}\left(I-P_{k}\right)\right) \xi, \xi\right\rangle\right) \\
& \geqq\left\|P_{0} \xi\right\| \alpha\left(\left\langle P_{0} \xi, \xi\right\rangle-\sum_{k=1}^{n}\left\langle P_{k} \xi, \xi\right\rangle\right) \\
& \geqq\left\|P_{0} \xi\right\| \alpha\left(\phi\left(P_{0}\right)-\sum_{k=1}^{\infty} \phi\left(P_{k}\right)\right) .
\end{aligned}
$$

Hence

$$
\begin{aligned}
& \left\langle P_{0} \eta, \xi\right\rangle=\left\langle\eta, P_{0} \xi\right\rangle=\lim _{n \rightarrow \infty}\left\langle\left(\prod_{k=1}^{n}\left(I-P_{k}\right)\right) \xi, P_{0} \xi\right\rangle \\
& \quad \geqq\left\|P_{0} \xi\right\| \alpha\left(\phi\left(P_{0}\right)-\sum_{k=1}^{\infty} \phi\left(P_{k}\right)\right) \\
& \quad>0 .
\end{aligned}
$$

The Cauchy-Schwarz inequality now ensures that $P_{0} \eta \neq 0$. To complete the proof of our special case of the theorem, it only remains to take $x=\left\|P_{0} \eta\right\|^{-1} P_{0} \eta$.

To handle the general case, we first apply 2.2 to construct a sequence $\left(\xi_{k}\right)_{k \geqq 1}$ in $H$ such that $\sum_{k=1}^{\infty}\left\|\xi_{k}\right\|^{2}$ is convergent and $\phi(R)=$ $\sum_{k=1}^{\infty}\left\langle R \xi_{k}, \xi_{k}\right\rangle$ for each $R$ in $\mathscr{L}(H)$. Let $H_{\infty}$ be the direct sum of a sequence of copies of $H$, with the standard scalar product, and let $\boldsymbol{\xi}=\left(\xi_{k}\right)_{k \geq 1}$. For each projection $P$ on $H$ and each $\boldsymbol{x}=\left(x_{k}\right)_{k \geqq 1}$ in $H_{\infty}$, define $\boldsymbol{P} \boldsymbol{x}=\left(\boldsymbol{P} x_{k}\right)_{k \geq 1}$. Then $\boldsymbol{P}$ is a projection on $H_{\infty}$ and $\langle\boldsymbol{P} \boldsymbol{\xi}, \boldsymbol{\xi}\rangle=$ $\sum_{k=1}^{\infty}\left\langle P \xi_{k}, \xi_{k}\right\rangle=\phi(P)$. Thus

$$
\sum_{n=1}^{\infty}\left\langle\boldsymbol{P}_{n} \xi, \boldsymbol{\xi}\right\rangle=\sum_{n=1}^{\infty} \phi\left(P_{n}\right)\left\langle\phi\left(P_{0}\right)=\left\langle\boldsymbol{P}_{0} \xi, \boldsymbol{\xi}\right\rangle .\right.
$$

As $\left(\boldsymbol{P}_{n}\right)_{n \geq 0}$ is a sequence of pairwise commuting projections on $H_{\infty}$, the first part of the proof shows that there exists $\boldsymbol{x}=\left(x_{k}\right)_{k \geqq 1}$ in $H_{\infty}$ such that $\sum_{k=1}^{\infty}\left\|x_{k}\right\|^{2}=\|\boldsymbol{x}\|^{2}=1, \boldsymbol{P}_{0} \boldsymbol{x}=\boldsymbol{x}$, and $\boldsymbol{P}_{n} \boldsymbol{x}=(0)$ for each $n \geqq 1$. With $k$ chosen so that $\left\|x_{k}\right\| \neq 0$, we complete the proof by setting $x=\left\|x_{k}\right\|^{-1} x_{k}$.

We end with the analogue of the fourth postulate for the BishopCheng integral [2, p. 2]. This leaves undiscussed only the third postulate, whose analogue is the unnecessary restriction that $\|\phi\|$ be positive.

THEOREM 3.4. Let $\phi$ be a countably additive, positive linear functional on $\mathscr{L}(H)$, and let $A$ be a selfadjoint operator on $H$. Then $\lim _{n \rightarrow \infty} \phi(A \wedge n I)=\phi(A)$ and $\lim _{n \rightarrow \infty} \phi\left(|A| \wedge n^{-1} I\right)=0$.

Here, $A \wedge n I$ and $|A| \wedge n^{-1} I$ are to be interpreted by means of the functional calculus for $A$. The proof of 3.7 is a fairly straight- 
forward application of that calculus, and is left to the reader.

\section{REFERENCES}

1. E. A. Bishop, Foundations of Constructive Analysis, McGraw-Hill, New York, 1967. 2. E. A. Bishop and H. Cheng, Constructive measure theory, Amer. Math. Soc. Memoirs, 116 (1972).

[3] D. S. Bridges, Constructive Functional Analysis, Research Notes in Mathematics, 28, Pitman, London, 1979.

4. - On weak operator compactness of the unit ball of $\mathscr{L}(H)$, Z. Math. Logik Grundlagen Math., Bd 24 (1978), 493-494.

5. S. Gudder and J-P. Marchand, Noncommutative probability on von Neumann algebras, J. Math. Phys., 13 (1972), 799-806.

6. S. Sakai, $C^{*}$-algebras and $W^{*}$-algebras, Springer Verlag, Berlin, 1971.

Received June 8, 1979 and in revised form July 9, 1980.

The University College at Buckingham

Buckingham, England 



\section{PACIFIC JOURNAL OF MATHEMATICS}

\section{EDITORS}

DONALD BABBITT (Managing Editor)

University of Galifornia

Los Angeles, California 90024

Hugo RossI

University of Utah

Salt Lake City, UT 84112

C. C. MOORE AND ANDREW OGG

University of California

Berkeley, CA 94720
J. DugundJI

Department of Mathematics University of Southern California Los Angeles, California 90007

R. FinN and J. Milgram Stanford University Stanford, California 94305

\section{ASSOCIATE EDITORS}

R. ARENS

E. F. BeCKenbaCh

B. H. NEUManN

F. WOLF

K. YosHIDA

\section{SUPPORTING INSTITUTIONS}

UNIVERSITY OF ARIZONA

UNIVERSITY OF BRITISH COLUMBIA

CALIFORNIA INSTITUTE OF TECHNOLOGY

UNIVERSITY OF CALIFORNIA

MONTANA STATE UNIVERSITY

UNIVERSITY OF NEVADA, RENO

NEW MEXICO STATE UNIVERSITY

OREGON STATE UNIVERSITY
UNIVERSITY OF OREGON

UNIVERSITY OF SOUTHERN CALIFONIA

STANFORD UNIVERSITY

UNIVERSITY OF HAWAII

UNIVERSITY OF TOKYO

UNIVERSITY OF UTAH

WASHINGTON STATE UNIVERSITY

UNIVERSITY OF WASHINGTON 


\section{Pacific Journal of Mathematics}

\section{Vol. 95, No. $1 \quad$ September, 1981}

John Allen Beachy and William David Blair, On rings with bounded

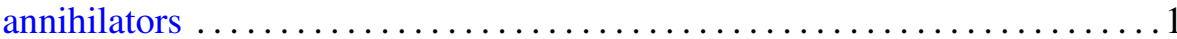

Douglas S. Bridges, A constructive look at positive linear functionals on

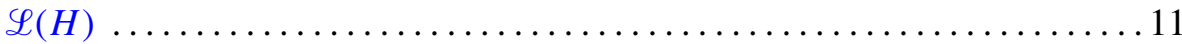

Muneo Chō and Makoto Takaguchi, Boundary points of joint numerical ranges

W. J. Cramer and William O. Ray, Solvability of nonlinear operator equations

Lester Eli Dubins and Gideon Schwarz, Equidiscontinuity of

Borsuk-Ulam functions

Maria Fragoulopoulou, Spaces of representations and enveloping 1.m.c.

*-algebras

Robert F. Geitz and J. Jerry Uhl, Jr., Vector-valued functions as families of scalar-valued functions

Ross Geoghegan, The homomorphism on fundamental group induced by a homotopy idempotent having essential fixed points

Ross Geoghegan, Splitting homotopy idempotents which have essential fixed points

Paul Jacob Koosis, Entire functions of exponential type as multipliers for

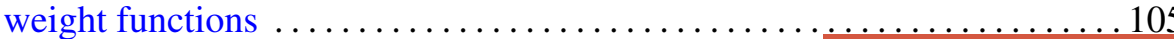

David London, Monotonicity of permanents of certain doubly stochastic matrices

Howard J. Marcum, Two results on cofibers

Giancarlo Mauceri, Zonal multipliers on the Heisenberg group

Edward Wilfred Odell, Jr. and Y. Sternfeld, A fixed point theorem in $c_{0} \quad \ldots 161$

Bernt Karsten Oksendal, Brownian motion and sets of harmonic measure zero

Andrew Douglas Pollington, The Hausdorff dimension of a set of normal numbers

Joe Repka, Base change lifting and Galois invariance ...

Gerald Suchan, Concerning the minimum of permanents on doubly stochastic circulants

Jun-ichi Tanaka, On isometries of Hardy spaces on compact abelian groups

Aaron R. Todd, Quasiregular, pseudocomplete, and Baire spaces 